\title{
Arabidopsis SFAR4 is a novel GDSL-type esterase involved in fatty acid degradation and glucose tolerance
}

\author{
Li-Min Huang ${ }^{1,6}$, Chia-Ping Lai ${ }^{2^{*}}$, Long-Fang O. Chen ${ }^{1,3}$, Ming-Tsair Chan ${ }^{1,5,6}$ and Jei-Fu Shaw ${ }^{1,4,7^{*}}$
}

\begin{abstract}
Background: SFARs (seed fatty acid reducers) belonging to the GDSL lipases/esterases family have been reported to reduce fatty acid storage and composition in mature Arabidopsis seeds. GDSL lipases/esterases are hydrolytic enzymes that possess multifunctional properties, such as broad substrate specificity, regiospecificity, and stereoselectivity. Studies on the physiological functions and biochemical characteristics of GDSL lipases/esterases in plants are limited, so it is important to elucidate the molecular functions of GDSL-type genes.

Results: We found that SFAR4 (At3g48460), a fatty acid reducer belonging to the Arabidopsis GDSL lipases/esterases family, was intensely expressed in embryo protrusion, early seedlings, and pollen. The characterization of recombinant SFAR4 protein indicated that it has short-length $p$-nitrophenyl esterase activity. In addition, SFAR4 enhanced the expression of genes involved in fatty acid metabolism during seed germination and seedling development. SFAR4 elevated the expression of COMATOSE, which transports fatty acids into peroxisomes, and of LACS6 and LACS7, which deliver long-chain acetyl-CoA for $\beta$-oxidation. Furthermore, SFAR4 increased the transcription of PED 1 and PNC1, which function in importing peroxisomal ATP required for fatty acid degradation. SFAR4 has another function on tolerance to high glucose concentrations but had no significant effects on the expression of the glucose sensor HXK1.
\end{abstract}

Conclusions: The results demonstrated that SFAR4 is a GDSL-type esterase involved in fatty acid metabolism during post-germination and seedling development in Arabidopsis. We suggested that SFAR4 plays an important role in fatty acid degradation, thus reducing the fatty acid content.

Keywords: SFAR, GDSL, Arabidopsis, Glucose tolerance

\section{Background}

GDSL-type esterases/lipases, a new subclass of lipolytic enzymes, are hydrolytic enzymes with multifunctional properties such as broad substrate specificity, regiospecificity, and stereoselectivity. They are widely distributed in bacteria and plants. GDSL lipolytic enzymes possess a distinct GDSL motif with a serine-containing active site near the N-terminus; hence, they differ from classical lipolytic enzymes with a $\mathrm{G} \times \mathrm{S} \times \mathrm{G}$ motif near the central

\footnotetext{
*Correspondence: glbhouse@yahoo.com.tw; shawjf@isu.edu.tw

2 Department of Food and Beverage Management, Far East University, No.

49, Zhonghua Rd., Xinshi Dist., Tainan City 74448, Taiwan

${ }^{4}$ Department of Biological Science and Technology, I-Shou University, No.

1, Sec. 1, Syuecheng Rd., Dashu District, Kaohsiung City 84001, Taiwan

Full list of author information is available at the end of the article
}

conserved domain (Upton and Buckley 1995). Studies have illustrated that GDSL lipolytic enzymes have flexible activities, including thioesterase, protease, arylesterase, and lysophospholipase activities (Akoh et al. 2004; Molgaard et al. 2000; Li et al. 2000; Dalrymple et al. 1997). GDSL-type esterases/lipases have also been detected in various plant species, including Arabidopsis, rice, and maize, and they have been implicated in plant development, morphogenesis, and defense responses (Brick et al. 1995). Several GDSL lipase/esterases in various plant species possess substrate specificity. BnSCE3/BnLIP2 from Brassica napus L. has been reported as a sinapine esterase that strongly expressed during seed germination and that functions in both germination and morphogenesis (Ling et al. 2006; Clauss et al. 2008, 2011). An

\section{垈 Springer}


acetyl-CoA carboxylase gene, $A m G D S H 1$, was cloned in black-grass (Alopecurus myosuroides), and the characterization of the purified protein revealed that it was a carboxyesterase that activated the hydrolysis of the herbicide aryloxyphenoxypropionate (AOPP) (Cummins and Edwards 2004). Pepper CaGLIP1 exhibited various activities, such as the hydrolysis of long-chain and short-chain p-nitrophenyl esters (Hong et al. 2008). A GDSL lipase/ esterase isolated from sunflower (Helianthus annuus L.) seeds exhibited fatty acyl-ester hydrolase activity (Beisson et al. 1997). So far, plant GDSL-type esterases/lipases have not been proven to exhibit any lipase activity during seed germination or post-germinative growth or to mediate the relationship between stress and signaling pathway in Arabidopsis.

Gibberellic acid (GA) is essential for numerous aspects of plant development, such as seed germination, leaf expansion, stem elongation, flowering, and seed development (Richards et al. 2001; Sun and Gubler 2004). The DELLA proteins (RGA, GAI, RGL1, RGL2, and RGL3), comprising a class of GA signaling repressors, possess a highly conserved motif in their N-terminal domain in Arabidopsis. The presumptive DELLA-regulated genes involved in seed germination are highly distinct from those involved in flower development. An observation of the transcriptomes of DELLA mutants suggested that GA-mediated seed germination and flower development are distinctly controlled by DELLA-dependent transcriptomes (Cao et al. 2006). Recently, genes encoding several GDSL proteins, named SFAR genes, which are genes downregulated by DELLAs, have been reported to reduce fatty acid storage and composition in mature Arabidopsis seeds and to enhance tolerance to glucose stress during the seed germination (Chen et al. 2012). Among these genes, SFAR4 has been demonstrated to reduce the total fatty acid content and change the composition of unsaturated fatty acids in storage seeds. Transgenic plants with overexpressed SFAR4 exhibited tolerance to glucose stress and a higher germination rate (Chen et al. 2012). The relationship between glucose stress and SFAR genes in germinating seeds or during post-germinative seedling growth remains unknown. The mechanism underlying SFAR4-induced insensitivity to glucose in seeds or seedlings has not yet been elucidated.

Seed germination is associated with the degradation and mobilization of accumulated components in mature seeds (Bewley 1997). During germination, the Arabidopsis storage lipids are converted to sucrose through the glyoxylate cycle. Sucrose synthesis and the peroxisomal pathways are most crucial to post-germinative growth (Penfield et al. 2004; Holdsworth et al. 2008; Footitt et al. 2006). Sucrose stored in vacuoles or converted into glucose or UDP-glucose and fructose is essential as an energy source and for signaling during seed germination (Koch 2004). However, a high sugar concentration delays seed germination and inhibits cotyledon expansion and greening, true leaf formation, and root growth of Arabidopsis seedlings (Rolland et al. 2006). Exogenous glucose significantly retards the mobilization of seed storage lipids in germinating Arabidopsis seeds. Critical metabolic transition of the primary sugar source results in a loss of sensitivity to the inhibitory effect of glucose on lipid breakdown and early seedling development (To et al. 2002). Two genes involved in the glyoxylate cycle and playing a role in lipid metabolism, malate synthase $(M S)$ and isocitrate lyase (ICL), have been reported to be negatively regulated by sugars. Using glyoxylate cycle mutants as a paradigms also reveals that they are resistant to the inhibitory effects of exogenous glucose on storage seed lipid breakdown in germinating seeds (Graham et al. 1994). Products of fatty acid catabolism can pass from the peroxisome to the mitochondrion independently of the glyoxylate cycle (Eastmond et al. 2000). Comprehensive metabolic profiling studies have suggested that Arabidopsis seed germination efficiency is not only affected by the accumulation of reserves during seed development or by their mobilization during seed germination but also by additional, as yet unknown factors (Fait et al. 2006).

Hayashi et al. used ped mutants, which are necessary to maintain glyoxysomal function, to demonstrate that peroxisomal fatty acid $\beta$-oxidation plays an important role in producing sucrose from storage lipids during germination (Hayashi et al. 1998). Studies on the deficiency of 3-ketoacyl-CoA thiolase, PED1, have suggested that peroxisomal $\beta$-oxidation is essential for post-germinative growth in Arabidopsis (Poirier et al. 1999; Hayashi et al. 1998). During post-germinative growth, peroxisomal $\beta$-oxidation of fatty acids releases storage triacylglycerol to produce acetyl-CoA and converts it to sucrose as a source of metabolic energy and carbon skeletons. Prior to $\beta$-oxidation, fatty acids are transported into peroxisomes by CTS (Footitt et al. 2002), and acyl-CoA substrates for $\beta$-oxidation are delivered by peroxisomal long-chain acyl-CoA synthetases (LACS6 and LACS7) (Fulda et al. 2004). Both steps are required for post-germinative storage oil breakdown for successful seedling establishment. Recently, repressing peroxisomal adenine nucleotide carriers PNC1 and PNC2 were been found to impair peroxisomal ATP import, which inhibits fatty acid breakdown during early seedling growth (Linka et al. 2008). However, those studies indicate that $\beta$-oxidation of fatty acids is not required for Arabidopsis germination (Hayashi et al. 1998; Poirier et al. 1999; Fulda et al. 2004). Because fatty acids hydrolyzed from storage lipids are converted to CoA thioesters, storage lipid breakdown plays an 
important role during germination in oilseed plants (Graham and Eastmond 2002).

Exogenous sucrose delays lipid breakdown in Arabidopsis seedlings (Eastmond et al. 2000), and high sugar concentrations repress seed germination and seedling growth. However, the signal transduction pathways involved in responses to sugar are not fully known. Hexokinase (HXK) signaling may inhibit the mobilization of lipids and other storage compounds, thus preventing germination (Pego et al. 1999; Smeekens 2000). HXK and SNF1-related protein kinases are postulated to control several sugar-regulated processes. Hexokinase 1 (HXK1) is a sugar sensor with both signaling and metabolic functions in plants (Jang et al. 1997). In the sugar signaling pathway, HXK1 is a glucose sensor that modulates gene expression and multiple plant hormone-signaling pathways underlying sugar sensing and signaling in plants (Pego et al. 1999; Jang et al. 1997). Limited information exists regarding the role of GDSL-type genes in determining lipid metabolic cellular signaling during seed germination and post-germinative growth. It therefore becomes important to study the role of GDSL-type genes involved in lipid metabolic pathways and the mechanisms underlying sugar sensitivity.

This study provides evidence that SFAR4 plays a crucial role in the lipid metabolic process and induction of tolerance to glucose stress through a glucose sensor-independent pathway during seed germination in Arabidopsis. Furthermore, the characterization of the recombinant SFAR4 reveals that it is a novel GDSL-type esterase. These observations suggest that SFAR4 plays an important physiological role in seed germination, seedling growth, and susceptibility to sugar.

\section{Methods}

\section{Sequence and motif analysis of SFAR4}

Using the BLAST program to align the EST database for the SFAR4 (At3g48460) sequence, full-length SFAR4 cDNA was identified. Specific primers were designed to amplify the full-length SFAR4 cDNA. The PCR products were cloned and sequenced. The sequence was subjected to BLAST analysis using the National Center for Biotechnology Information (NCBI) Arabidopsis genomic DNA database to identify the gene structure of SFAR4. The GDSL motif and catalytic triad sites of SFAR4 were characterized according to the SFAR4 sequence alignments to Arabidopsis GLIP1 and the typical GDSL-lipase-like enzymes within the MEGA 4.0 version software. The signal peptide and location of cleavage sites within the amino acid sequences were predicted using the SignalP 4.1 server. Motif analysis was performed with the Multiple Em for Motif Elicitation (MEME) motif search tool.

\section{Construction and transformation of SFAR4 in a yeast expression system}

cDNA fragments of SFAR4 were amplified and cloned into a pGAPZ $\alpha \mathrm{C}$ vector and expressed in Pichia pastoris SMD168H (Invitrogen). P. pastoris was cultured in YPD media ( $2 \%$ peptone, $1 \%$ yeast extract, $2 \%$ glucose, and $1.5 \%$ agar, $\mathrm{pH}$ 7.0). Plasmids were linearized with the restriction enzymes AvrII or $B s p \mathrm{HI}$ and transformed into P. pastoris SMD168H by electroporation for $5 \mathrm{~ms}$ (Chang et al. 2006). High-voltage pulses $(1.5 \mathrm{kV})$ were introduced to $100-\mu \mathrm{L}$ samples in $0.2-\mathrm{cm}$ electrode gap cuvettes using a BTX Electro Cell Manipulator apparatus. Transformed P. pastoris colonies of constructions were plated on YPD supplemented with zeocin (100 ng L ${ }^{-1}$, Invitrogen) for colony selection.

\section{Lipase or esterase activity plate assays, purification, and specific activity analysis}

Recombinant SFAR4 of $P$. pastoris transformants were screened using a $1.5 \%$ tributyrin emulsion agar or $2 \%$ Tween-20 plates. The tributyrin solution was sonicated to a milky emulsion before adding the autoclaved YPD media. Tween-20 plates were prepared by using modified YPD media (YPD media supplemented with $0.5 \% \mathrm{CaCl}_{2}$, $2 \%$ Tween-20, pH 7.0) (Cardenas et al. 2001). Lipase activity for tributyrin emulsion was determined by the appearance of clear rings around each colony, whereas for Tween-20, lipase activity was based on the formation of a white calcium precipitate. Colonies showing hydrolytic and lipolytic activity with respect to Tween-20 and tributyrin were isolated and further cultured and purified with HisTrap Ni Sepharose.

Enzyme activity was measured according to Chang et al. (2006). Lipase/esterase activity was assayed by using a spectrophotometer. The hydrolysis of $p$-nitrophenyl esters was conducted at $30^{\circ} \mathrm{C}$ in $500 \mu \mathrm{L}$ of $50 \mathrm{mM}$ Good's buffer (50 mM each of bicine, CAPS, sodium acetate, and bis-Tris propane), $\mathrm{pH} 7.0$, containing $0.24 \%$ Triton $\mathrm{X}-100$ and $5 \mathrm{mM}$ p-nitrophenyl esters. The increase in absorbance was recorded for $10 \mathrm{~min}$ at a wavelength of $348 \mathrm{~nm}$. One unit of activity was defined as the quantity of enzyme necessary to release $1 \mu \mathrm{mol}$ of $p$-nitrophenol per minute. Each experiment was conducted in triplicate. Error bars represented standard deviation (SD).

\section{Antibody preparation}

For antibody preparation, the recombinant SFAR4 protein devoid of the putative signal peptide was amplified and cloned into a pET23a (+) vector and expressed in E. coli BL21 (DE3) competent cells (Novagen). The BL21 (DE3) transformants were cultured in LB media supplemented with ampicillin $\left(50 \mathrm{ng} \mathrm{L^{-1 }}\right)$ at $37{ }^{\circ} \mathrm{C}$ overnight, 
then refreshed for $2 \mathrm{~h}$ and induced with $0.1 \mathrm{mM}$ of IPTG for $6 \mathrm{~h}$ at $37^{\circ} \mathrm{C}$. The cultures were centrifuged to collect bacterial cells, which were then re-suspended in phosphate buffer and sonicated with a Misonix Sonicator XL2020. The sonication condition was $5 \mathrm{~s}$ on/off with $50-60 \%$ amplitude on ice for $5 \mathrm{~min}$ with a $3-\mathrm{mm}$ probe. Because of inclusion body production, the cell lysate was centrifuged at $20,000 \times g$ for $20 \mathrm{~min}$ at $4{ }^{\circ} \mathrm{C}$ and the pellet was collected. The pellet was washed twice with wash buffer ( $5 \mathrm{mM}$ imidazole, $0.5 \mathrm{M} \mathrm{NaCl}, 20 \mathrm{mM}$ phosphate buffer, $\mathrm{pH} 7.4$ and $0.5 \%$ Triton X-100), resuspended in buffer ( $8 \mathrm{M}$ urea, $0.5 \mathrm{M} \mathrm{NaCl}, 20 \mathrm{mM}$ phosphate buffer, $\mathrm{pH}$ 8.0), and homogenized with a tissue homogenizer. The supernatants following centrifugation $(47,800 \times g$, $20 \mathrm{~min}, 4{ }^{\circ} \mathrm{C}$ ) were recovered and further purified. The soluble recombinant SFAR4s were purified through a HisTrap affinity column (GE Healthcare Life Sciences). The purified proteins were separated on the SDS-polyacrylamide gel (SDS-PAGE) and stained with Coomassie Brilliant Blue R-250 to confirm the molecular size and the purity. Purified recombinant SFAR4 protein was used for antiserum production. A rabbit polyclonal antibody specific to recombinant SFAR4 was produced by Allbio Science, Inc. (Taichung City, Taiwan), using a standard 70-day rabbit immunization protocol for rabbit polyclonal antibody production.

\section{Plant materials and growth conditions}

To study the biological functions of SFAR4 genes, we searched for putative SFAR4 T-DNA insertional mutants from the T-DNA Express database of the SALK Institute Genome Analysis Laboratory (SIGnAL; http://signal. salk.edu/cgi-bin/tdnaexpress) and identified their exact integrated positions. Arabidopsis plants with ecotypes Col-0 were used as wild types for the phenotypic evaluation of plant growth and development. All of the Arabidopsis seeds underwent imbibition for 2 days and were then grown in soil at $22{ }^{\circ} \mathrm{C}$ under a 16 -h light/8-h dark cycle. For aseptic growth, seeds were surface-sterilized and sown on Murashige and Skoog (MS) medium (Duchefa) and solidified with $0.7 \%$ phytoagar (Duchefa). After sowing, the plates were incubated at $4{ }^{\circ} \mathrm{C}$ in the dark for 2 days and subsequently transferred to the growth chamber at $22{ }^{\circ} \mathrm{C}$ under 16 -h light/8-h dark cycle. For osmotic and salt stress studies, Arabidopsis plants were surface-sterilized and then seeded on MS media plates supplemented with or without salts $(\mathrm{NaCl}, \mathrm{KCl}$, or $\mathrm{LiCl})$, mannitol, and glucose at $\mathrm{pH}$ 5.7. Growth conditions were as described above. All data are the mean value of at least 50 plants, and these experiments were three replicates, obtaining similar values in each experiment. Data values of cotyledon expansion and greening for seedlings were statistically analyzed using the Student's $t$ test.

\section{Plant transformation}

Transgenic plants were generated by Agrobacterium tumefaciens-mediated transformation in Arabidopsis. Full-length SFAR4 was amplified and constructed into pCAMBIA1300-modified vectors that contained the constitutive cauliflower mosaic virus (CaMV) $35 \mathrm{~S}$ promoter. Then, the constructs were transformed into A. tumefaciens LBA4404 by electroporation. The transformants were screened with the neo gene (kanamycin-resistant) and introduced into plants by floral dip transformation (Clough and Bent 1998). The seeds from the transfected plants were harvested, surface-sterilized, and plated on a hygromycin selection media. Positive seedlings with 4 to 6 adult leaves were then transplanted and maintained in the greenhouse. Homozygous lines were identified in the next generation.

To study the expression pattern of SFAR4, Pro $_{\text {SFAR }}$ :GUS transgenic plants with the $\beta$-glucuronidase (GUS) reporter gene under the control of the SFAR4 promoter were cloned into the pBI101 plasmid, then generated by Agrobacterium tumefaciens-mediated genetic transformation as previously described. The length of SFAR4 promoter is $2.1 \mathrm{~kb}$ upstream from the transcription start site. Col-0 wild-type plants were used for the construction of Pro ${ }_{\text {SFAR }}$ : GUS transgenic plants. An efficient and largely genotype-independent transformation of Arabidopsis was established based on the use of the neo gene as a selectable marker gene. The seeds from the transfected plants were harvested, surface-sterilized, and plated on the kanamycin selection media. Homozygous lines were identified in the next generation and the expression of GUS reporter gene was monitored by the GUS staining method. Staining for GUS activity was performed according to a modified protocol as Schoof et al. (2000) described. Plant material was prefixed in $90 \%$ acetone for $20 \mathrm{~min}$ at room temperature, rinsed in staining buffer without 5-bromo-4-chloro-3-indolyl glucuronide (X-Gluc), and infiltrated with staining solution (50 mM NaPO, pH 7.2; 2 mM potassium-ferrocyanide; $2 \mathrm{mM}$ potassium-ferricyanide; $0.2 \%$ Triton X-100; $2 \mathrm{mM}$ $\mathrm{X}$-Gluc) under vacuum on ice for $15 \mathrm{~min}$ and incubated at $37{ }^{\circ} \mathrm{C}$ for $3-8 \mathrm{~h}$. After dehydration in an ethanol series up to $70 \% \mathrm{EtOH}$, tissue was stored at room temperature until capturing the images. Photographs were taken with a Zeiss Microscope camera. To analyze the expression pattern of SFAR4, at least 10 independent lines of Pro $_{\text {SFAR } 4}$ :GUS $\mathrm{T}_{3}$ homozygous were selected for the GUS staining experiments. In most cases, independent transgenic lines showed similar GUS activity patterns. However, examples of single lines that showed an expression pattern distinct from other lines were also identified. We reasoned that such lines may have the transgene inserted at a genomic site that aberrantly influenced expression or 
may have experienced a mutation upon transformation. Thus, GUS expression analyses are shown only when at least five independent transgenic lines displayed similar GUS expression patterns.

\section{Isolation of genomic DNA and genomic PCR and Southern blot}

Correct T-DNA insertion lines were determined by using genomic PCR. Genomic DNA was isolated from individual plants as described by Winnepenninckx et al. (1995) and amplified by PCR reactions with a set of primers containing SFAR4 gene-specific primers and a T-DNA left border specific primer (Additional file 1: Table S1). PCR products were sequenced and the accurate insertional site of T-DNA was confirmed. These mutants were then determined the T-DNA insertional number by Southern blot.

DNA blot was conducted according to the procedure of Solanas and Escrich (1997) with minor modifications and detected by the DIG-labeling detection system. $10 \mu \mathrm{g}$ of genomic DNA was digested with a restriction enzyme and separated by $0.8 \%$ agarose gel electrophoresis, and then transferred onto a nylon membrane. The membrane was incubated with a blocking solution for $1 \mathrm{~h}$ and then hybridized to a DIG-labeled single-stranded DNA probe overnight at $60{ }^{\circ} \mathrm{C}$. Low stringency washes in $2 \times$ $\mathrm{SSC} / 0.1 \% \mathrm{SDS}$ for $15 \mathrm{~min}$ at $37^{\circ} \mathrm{C}$ were performed twice, followed by high stringency washes using $0.2 \times \mathrm{SSC} / 0.1 \%$ SDS at $68{ }^{\circ} \mathrm{C}$, following the DIG Application Manual from Roche (South San Francisco, CA, USA).

\section{RT-PCR, and quantitative real-time PCR and Statistical analysis}

RNA was isolated from plants by using the RNeasy plant mini kit (Qiagen), and M-MLV reverse transcriptase was employed to generate cDNA. For the reverse transcription reactions, $2 \mu \mathrm{g}$ of total RNA extracted from 14-dayold seedlings were incubated with $0.5 \mu \mathrm{g}$ oligo dT in a total of $15 \mu \mathrm{L}$ at $70{ }^{\circ} \mathrm{C}$ for $5 \mathrm{~min}$. After chilling on ice for 5 min, components of the $1 \times$ RT buffer (Promega, Madison, WI, USA), $2.5 \mathrm{mM} \mathrm{MgCl}_{2}, 0.5 \mathrm{mM}$ dNTPs, 25 units of a ribonuclease inhibitor, and 200 units of Superscript RT were added as final concentrations and incubated at $42{ }^{\circ} \mathrm{C}$ for $60 \mathrm{~min}$. After incubating at $70{ }^{\circ} \mathrm{C}$ for $15 \mathrm{~min}$, four units of RNase $\mathrm{H}$ (Promega) were added and the reaction was incubated at $37{ }^{\circ} \mathrm{C}$ for $20 \mathrm{~min}$. To examine the expression pattern of $S F A R 4,1 \mu \mathrm{L}$ of cDNA from the $\mathrm{RT}$ reactions and the specific primer pair for SFAR4 were used to amplify the full-length cDNA of SFAR4 by PCR.

Quantitative real-time RT-PCR was performed by the CFX Connect ${ }^{\mathrm{TM}}$ Real-Time PCR Detection System (BioRad Inc.). Specific primers for each signal are shown in Additional file 1: Table S1. The melting curve was used to confirm the specificity of primers. Dissociation curves of each primer contained a single peak with no shoulders, and the agarose gels of the amplified product revealed single bands corresponding to the predicted amplicon length. All data are the mean value of three independent measures, and the error bars correspond to the standard error. Data values were statistically analyzed using the Student's $t$ test.

\section{Results and discussion}

\section{Identification and characterization of SFAR4}

SFAR4, as GDSL-like lipases/esterases, was localized on chromosome 3 and was comprised of 3 exons and 2 introns (Additional file 2: Fig. S1A). The full-length SFAR4 cDNA was 1143 bp in length and encoded a 381 -amino-acid protein (approximately $42.3 \mathrm{kDa}$ ). The GDSL motif and catalytic triad sites of the SFAR4 protein were characterized by aligning the SFAR4 sequence with the sequences of Arabidopsis GLIP1 and the typical GDSL-lipase-like enzymes using MEGA 4.0 version software. SFAR4 contained conserved motifs of typical GDSL lipase/esterase family proteins, which form the catalytic triad and oxyanion hole (Akoh et al. 2004). Four conserved residues, Ser-Gly-Asn-His, were present in the conserved blocks I, II, III, and V, respectively. Hence, SFAR4 is an SGNH-hydrolase (a subgroup of GDSL esterases/lipases) that contains a conserved GDSL motif near the $\mathrm{N}$-terminus along with the predicted esterase/ lipase catalytic triad active sites, Ser45, Asp187, and His347 (Additional file 2: Fig. S1B). The phylogenetic analysis of SFAR4 protein and 24 plant GDSL esterases/ lipases (Additional file 3: Fig. S2) indicated that the evolutionary relationship of SFAR4 was homologous to that of BnSCE3/BnLIP2 in Brassica napus, myosuroides hydrolase (AmGDSH1) in the weed black-grass (Alopecurus myosuroides), acetylajmalan esterase (AAE) in Rauvolfia, and ARAB-1 in Arabidopsis (Ling et al. 2006; Cummins and Edwards 2004; Ruppert et al. 2005; Mikleusevic et al. 2009). We suggest that the molecular functions of SFAR4 maybe contain the esterase activity but the physiological function is still unclear.

From the ontological annotation of molecular functions of the TAIR gene, SFAR4 is a secreted hydrolase acting on ester bonds, is involved in the glycerol biosynthetic and lipid metabolic processes, and is located in the endomembrane system (https://www.arabidopsis.org/servlets/TairObject?id=40278\&type=locus). To characterize the specific activities of SFAR4, full-length SFAR4 cDNA was cloned into an expression vector and then overexpressed in yeast (Pichia pastoris). However, analyses of the full-length recombinant SFAR4 protein revealed that it exhibited no esterase or lipase activity (data not shown). We presumed that SFAR4 might be processed 
or undergo post-translational modifications. Analysis of the full-length SFAR4 protein using the SignalP 4.1 server showed that SFAR4 possessed a signal peptide that targets the extracellular components (Additional file 2: Fig. S1). The signal peptide and location of cleavage sites in the amino acid sequences were predicted between residues 26 and 27 at the $\mathrm{N}$-terminus (Additional file 2: Fig. S1B). The results indicated that the full-length SFAR4 cDNA sequence encoded a precursor of SFAR4 with the signal peptide and that the precursor protein was further processed into a mature protein (355 amino acids) with a predicted molecular mass of $39.6 \mathrm{kDa}$. The SFAR4 cDNA without a putative signal sequence was then cloned into the pGAPZ $\alpha$ C vector (Invitrogen) and expressed in $P$. pastoris. The transformants with the recombinant SFAR4 protein without a putative signal peptide were selected by hydrolytic activity with Tween-20 and tributyrin (Fig. 1a, b). These transformants were further cultured and purified using HisTrap Ni Sepharose. The recombinant SFAR4 protein also showed esterase activity toward $\alpha$-naphthyl butyrate (Additional file 4: Fig. S3) in native gel analysis. The specific activity of the purified enzyme was $6.25 \mu \mathrm{M} \mathrm{min}{ }^{-1} \mathrm{mg}^{-1}$ protein with $p$-nitrophenyl butyrate as a substrate and with approximately 1.75 -fold purification (Table 1). The specific activity of the purified recombinant SFAR4 enzyme toward the $p$-nitrophenyl esters with 2- to 16-carbon acyl groups was analyzed. The results showed that SFAR4 protein is an esterase that favors esters with short acyl chains as substrates (Fig. 1c).

In this study, we found that recombinant SFAR4 protein expressed in E. coli was not active. However, the specific activity analysis of the recombinant SFAR4 protein expressed in yeast showed that it possessed hydrolase activity toward acyl esters (Fig. 1). The recombinant protein expressed in bacteria was deficient in post-translational modifications, whereas that expressed in Pichia pastoris underwent glycosylation modifications. The annotation in the UniPprot database (http://www. uniprot.org/uniprot/Q9STM6) predicted that SFAR4 possessed amino acid modifications in the form of glycosylation at residues 112, 140, and 158. Therefore, we suggested that SFAR4 proteins need post-translational modifications for their activities, such as amino acid glycosylation or correct folding.
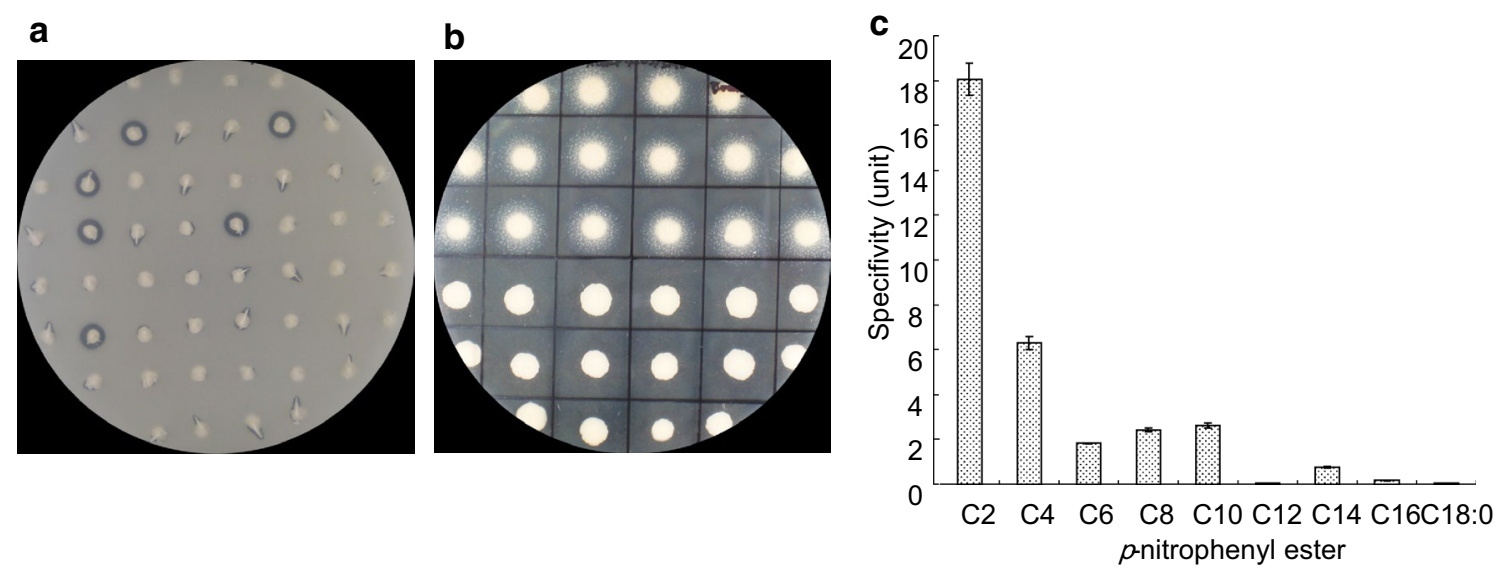

Fig. 1 Recombinant SFAR4 proteins for lipase/esterase activity analysis. a YPD agar plates supplemented with $1.5 \%$ tributyrin emulsion and secreted recombinant SFAR4 that hydrolyzes tributyrin to produce clear zones. b YPD supplemented with $0.5 \% \mathrm{CaCl}_{2}$ and $2 \%$ Tween- 20 , with hydrolytic activity indicated the formation of a white calcium precipitate. Transformed SFAR4 yeast colonies were grown on agar plates for 5 days. c Substrate specificity was determined by measuring activities for acyl $p$-nitrophenyl esters at $5 \mathrm{mM}$ concentrations and monitoring the release of p-nitrophenol photometrically at $348 \mathrm{~nm}$. These assays were performed using $50 \mathrm{mM}$ Good's buffer $(\mathrm{pH} 7.0)$ at $30^{\circ} \mathrm{C}$. One unit of enzyme is defined as the amount of enzyme that hydrolyzes $1 \mu \mathrm{mol}$ of the substrate per minute

Table 1 Purification of enzymes catalyzing the p-nitrophenyl butyrate reaction from Pichia pastoris

\begin{tabular}{lllllc}
\hline Purified step & Total protein $(\mathbf{m g})$ & $\begin{array}{l}\text { Total activity } \\
\left(\boldsymbol{\mu} \mathbf{~ m i n}^{-1}\right)\end{array}$ & Yield (\%) & $\begin{array}{l}\text { Specific activity }\left(\boldsymbol{\mu M} \mathbf{~ m i n}^{-\mathbf{1}}\right. \\
\mathbf{m g}^{-\mathbf{1}} \text { protein) }\end{array}$ & $\begin{array}{l}\text { Enrichment } \\
(\mathbf{f o l d})\end{array}$ \\
\hline Secreted medium (20 times concentrate) & 2.00 & 7.16 & 100 & 3.58 & 1 \\
HisTrap Sepharose & 0.15 & 0.94 & 13.10 & 6.25 & 1.75 \\
\hline
\end{tabular}




\section{Expression pattern of SFAR4}

The hormone GA plays a critical role in various processes of plant development, such as seed germination, leaf expansion, stem elongation, flowering, and seed development (Richards et al. 2001; Sun and Gubler 2004). It has been reported that DELLA proteins, which are GA signaling repressors, regulate the expression of numerous GDSL-type lipase/esterase genes during flower and seed development and germination (Cao et al. 2006). It has been demonstrated that five GDSL-type lipase/esterase genes, SFAR1-5, that were down-regulated by DELLA on DELLA mutant transcriptomes, are involved in reducing fatty acid storage and composition in mature Arabidopsis seeds (Chen et al. 2012). However, lipid hydrolysis is not essential for seed germination and seedling establishment (Kelly et al. 2011). Therefore, seed germination and development are distinctly controlled by DELLA-dependent transcriptomes. To analyze the diversity of DELLA-regulated GDSL-type lipase/esterase genes, the (MEME) program was used to elucidate the similarities among the conserved motifs of these proteins. Motifs among DELLA-downregulated GDSL-type enzymes with E-values from $1.02 \mathrm{e}-1356$ to $9.9 \mathrm{e}-164$ were found. Motifs 5, 9, 3, and 1 represent GDSL esterase/lipase conserved blocks I, II, III, and V, respectively. Most DELLA downregulated GDSL-type proteins with conserved motifs 6 and 7 differed significantly from SFAR4 (Additional file 5 : Fig. S4). These different domains of SFAR4 may result in distinct activities or functions that are involved in the response of plant physiology to environmental changes.

The sequencing study revealed that SFAR4 containing the classical motifs of GDSL-type lipases/esterases (Additional file 2: Fig. S1B), and a signal peptide may be targeted to the outside of the cell or to the membrane. The expression localization and stage of SFAR4 are likely to play a role in specific functions during plant growth. To investigate the SFAR4 expression pattern, transgenic plants carrying the GUS reporter gene under the control of the SFAR4 promoter, including the region $2.1 \mathrm{~kb}$ upstream of the transcription start sites, were generated through Agrobacterium tumefaciens-mediated genetic transformation. The results of the GUS reporter gene assay with X-Gluc staining indicated that SFAR4 is highly expressed during seed germination and early seedling growth (Fig. 2a-d). The expression of GUS in transgenic plants carrying Pro $_{\text {SFAR }}: G U S$ was intense during embryo protrusion and in cotyledons, early rosette leaves, and pollen (Fig. 2f). GUS expression was low or undetectable in the apical meristem, roots, mature siliques, dry seeds, and floral regions except in the pollen (Fig. 2c-h). The results of SFAR4 promoter triggers GUS gene expression were consistent with the observation of expression data collected from the Genevestigator microarray website (data not shown). These results indicate that the expression location and stage show the crucial role of SFAR4 in seed germination and early seedling development. Specially, intense GUS reporter gene staining during embryo protrusion showed that SFAR4 was highly expressed post-germination (Fig. 2a). These results indicate that SFAR4 plays a crucial role in seed germination and seedling growth.

\section{SFAR overexpression enhanced the germination rate and young seedling establishment during high glucose stress}

To study the biological functions of the SFAR4 gene, we searched the Salk Institute database for putative T-DNA insertion mutants of SFAR4 and obtained 2 alleles, sfar41 (salk_122440) and sfar4-2 (salk_008418), with T-DNA insertion in exon3 and exon1, respectively. From the SALK database, the T-DNA insertion sites in sfar4-1 and sfar4-2 were deduced to be in exon3 (1341 bp downstream of the ATG start codon) and exon1 (607 bp downstream of the ATG start codon). In this study, the results showed that the exact integrated sites were in exon3 (1567 bp downstream of the ATG start codon) and exon1 (605 bp downstream of the ATG start codon). The insertion site of salk_008418 is consistent with that deduced from the SALK database (Additional file 6: Fig. S5). A Southern blot analysis demonstrated that the sfart-1 and sfar4-2 lines contained single T-DNA insertions, and RTPCR revealed that the homozygous mutants of sfar4-1 and sfar4-2 harbored SFAR4 transcripts that underwent gene silencing. To investigate the role of SFAR4 genes in Arabidopsis, full-length cDNAs of transgenic plants expressing SFAR4 under the control of a CaMV 35S promoter were generated through Agrobacterium-mediated transformation. The homozygous overexpressor and knockout mutant lines were confirmed using western blot analysis. The results indicated that the SFAR4 overexpressors exhibited a higher SFAR4 expression level than did the wild-type plants, whereas expression in the knockout mutants (sfar4-1 and sfar4-2) was undetectable. The transgenic plants of two knockout mutants and overexpressors were further used to analyze the variant phenotypes (Additional file 6: Fig. S5).

The phenotypes of the sfar4 knockout mutants and overexpressors with various plant growth and development characteristics were examined. The growth and morphology of leaves, seeds, siliques, and flowers in the transgenic plants were compared with those in the wildtype plants under normal growth conditions. However, the phenotypes of the knockout mutants, sfar4-1 and sfar4-2, and the overexpressor plants, Pro $_{355}$ SFAR4\#10 and Pro $_{355}$ :SFAR4\#11, were similar to those of Col-0 wild-type plants. The phylogenetic analysis revealed that 


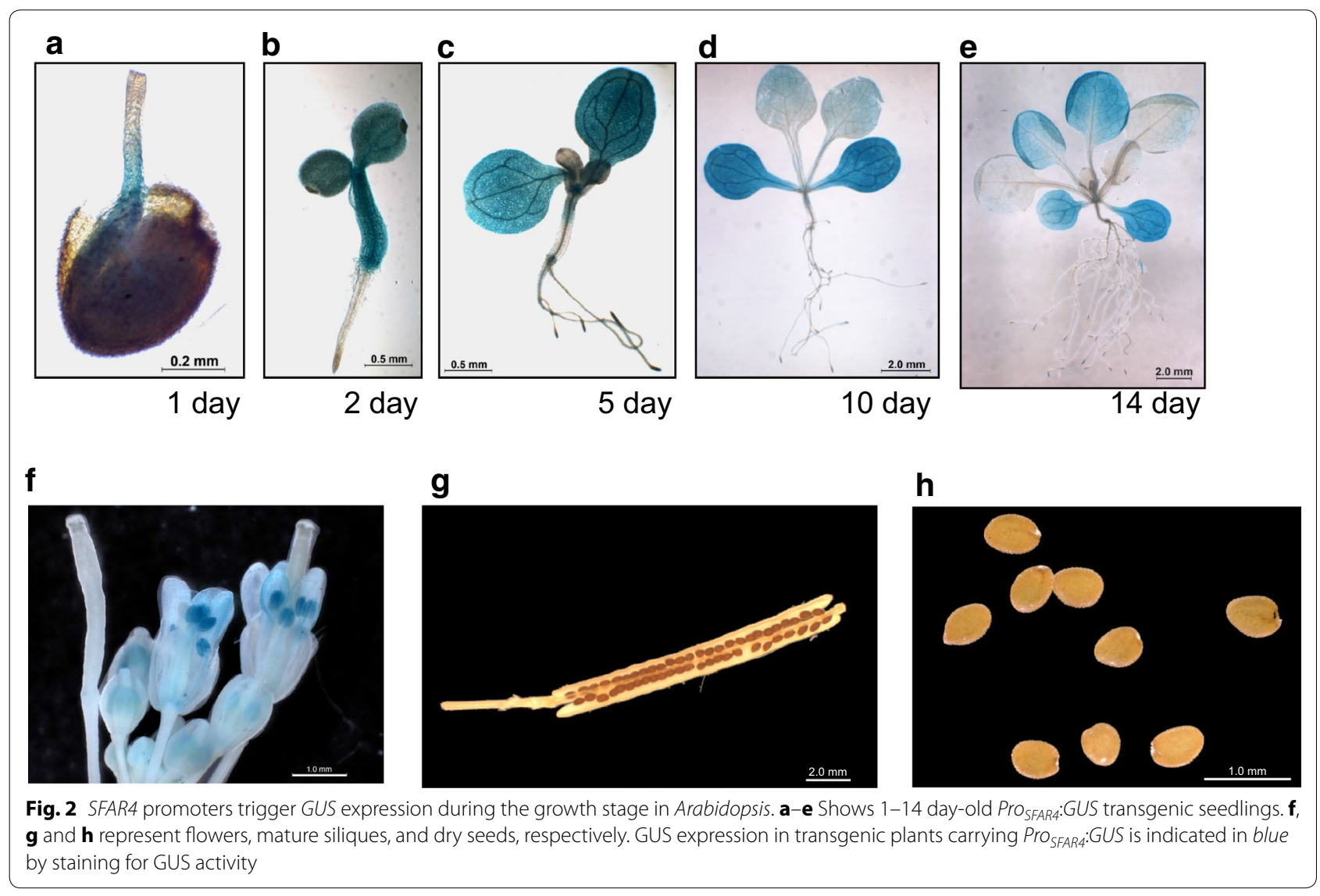

SFAR4 is closely related to these 24 GDSL-type proteins (Additional file 3: Fig. S2). According to the elucidated functions of these homologous genes (Additional file 7: Table S2), we suggested that SFAR4 might be involved in the stress response. Therefore, we examined the responses of sfar4 knockout mutants and Pro $_{355}$ :SFAR4 transgenic plants under salt $(\mathrm{LiCl}, \mathrm{KCl}$, and $\mathrm{NaCl})$ and osmotic (glucose and mannitol) stress. None of the mutants differed significantly from the wild-type plants in their germination and growth phenotypes on MS medium with or without salt during a 2-week observation period (Additional file 8: Fig. S6). Phenotype analysis of SFAR4 transgenic plants under osmotic (glucose and mannitol) stress showed that the germination rate of sfar4 knockout mutants was only $38 \%$ after 4 days in MS medium with $5 \%$ glucose (Fig. 3). By contrast, the germination rates in SFAR4 overexpressors $\left(\right.$ Pro $_{355}$ SFAR4 transgenic lines) and Col-0 wild-type plants in $5 \%$ glucose were 85 and $70 \%$, respectively (Fig. 3a). Both the expansion and greening of mutant cotyledons of sfar4-1 and sfar4-2 seedlings were more significantly inhibited by high glucose concentrations than those in wild-type seedlings (Fig. 3c). Compared with the wild-type plants, sfar4-1 and sfar4-2 were expanded only by approximately $55 \%$ and green cotyledons by approximately $20 \%$ in the presence of $5 \%$ glucose. However, the exogenous supply of $5 \%$ glucose affected cotyledon expansion in Pro $_{35 S}$ :SFAR4 transgenic plants by approximately $90 \%$ and in the Col-0 wild-type plants by approximately $85 \%$. This suggested that SFAR4 disruption enhanced the susceptibility to glucose, whereas SFAR4 overexpressors could partially mitigate this effect during seed germination. However, no statistically significant difference was observed between the transgenic and Col-0 wildtype plants during germination and cotyledon expansion or greening under mannitol treatment (Fig. 3a, c). These results suggest that hypersensitivity to glucose in the sfar4 knockout mutants was not caused by osmotic response (Fig. 3; Additional file 8: Fig. S6).

\section{Expression of HXK1 in SFAR4 transgenic plants during seed germination}

According the results shown in Fig. 3, SFAR4 overexpressors are insensitive to high glucose concentrations. These observations suggest that SFAR4 in Arabidopsis encodes a functional protein that might play a key role 

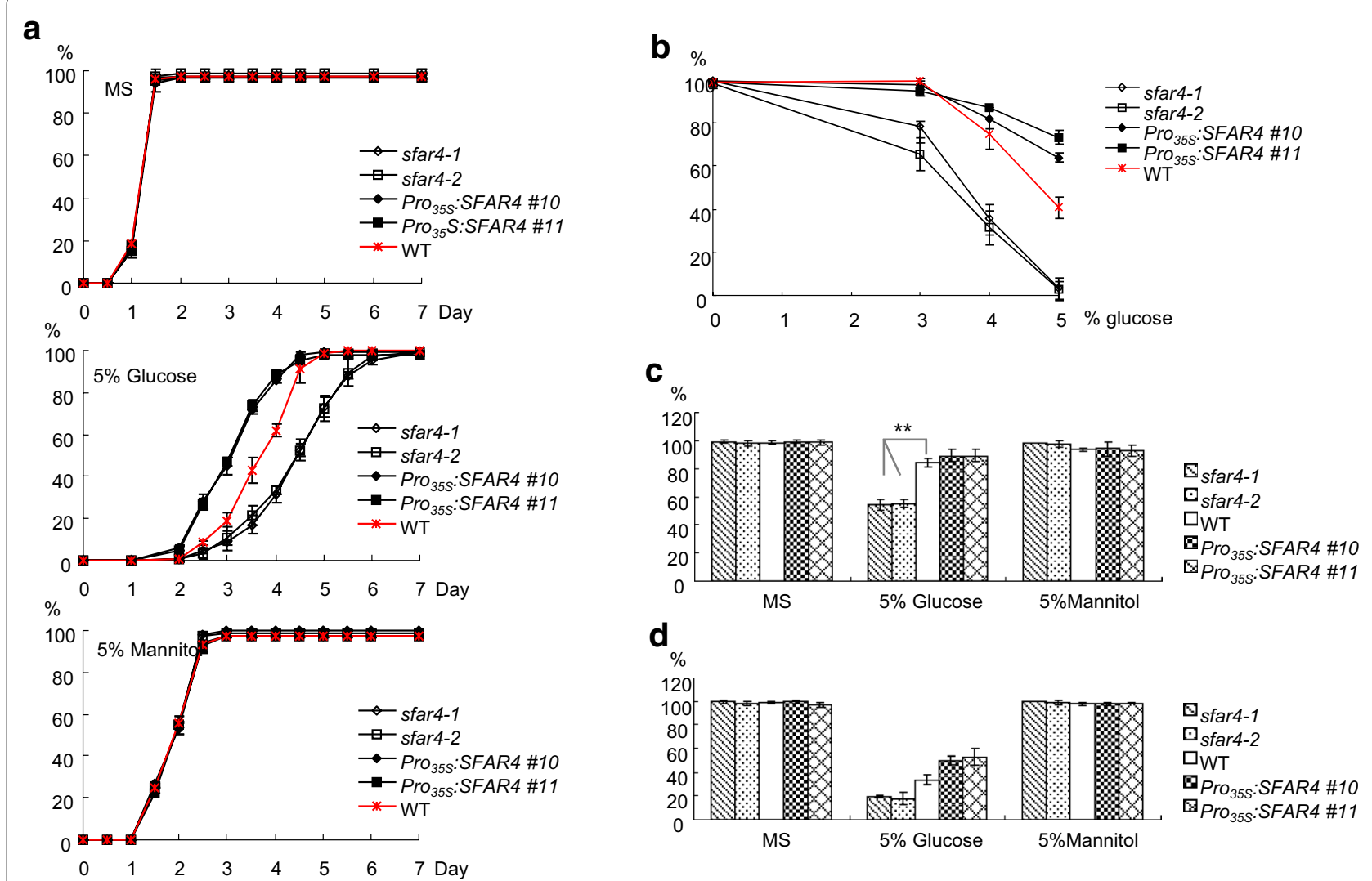

Fig. 3 Phenotype analysis of SFAR4 transgenic plants under osmotic (glucose and mannitol) stress. a Germination rates of SFAR4 transgenic plants in MS medium with or without $5 \%$ glucose or mannitol. Solid lines indicate Pro 355 :SFAR4 transgenic plants, whereas hollow lines indicate sfar4 knockout mutants. Wild-type (Col-0) is indicated by asterisks. Germination was defined as complete protrusion of the radicle. b Germination rates of SFAR4 transgenic plants in different concentrations of glucose at $84 \mathrm{~h}$. c, d The cotyledon expansion and greening of SFAR4 transgenic plants in MS medium plates with or without $5 \%$ glucose or $5 \%$ mannitol for 7 days. sfar4-1 and sfar $4-2$ represent knockout mutants, while Pro 355 :SFAR4 \#10 and \#11 indicate SFAR4 overexpressors. WT indicates wild type (Col-0). The percentage of the cotyledon expansion and greening seedlings were measured. Each value is the mean of three independent measures, and the error bars correspond to the standard error $(\mathrm{n}=50)$. Asterisks were used to indicate statistically significant difference compared with wild-type plants as determined by Student's $t$ test $\left({ }^{*} \mathrm{P}<0.05\right.$ and ${ }^{* *} \mathrm{P}<0.001$, respectively)

in glucose susceptibility and glucose metabolic pathway regulation in germinating seeds. Arabidopsis hexokinase 1 (HXK1) with the enzymatic function in glycolysis is suggested as a glucose sensor (Cho et al. 2006). A hxk1 mutant known to be glucose insensitive2 (gin2-1) eliminated seedling developmental arrest induced by $6 \%$ glucose (Moore et al. 2003), while HXK1 overexpressors resulted in reduced hypocotyl elongation in seedlings and reduced plant growth (Karve et al. 2012). Hence we investigated that the relative transcription levels of $H X K 1$ in SFAR4 transgenic plants compared with the WT (Col0 ) imbibed seeds (Im48hr) and post-germinated seedlings $(24 \mathrm{~h})$. The statistical analysis for Student's $t$ test indicated that $H X K 1$ expressions have no significantly difference between wild type, sfar4 knockout mutants and SFAR4 overexpressors. These results indicated that SFAR4 has no effect on HXK1 expression (Fig. 4). Therefore, we suggest that SFAR4-meidated glucose response is HXK1-independent pathway.

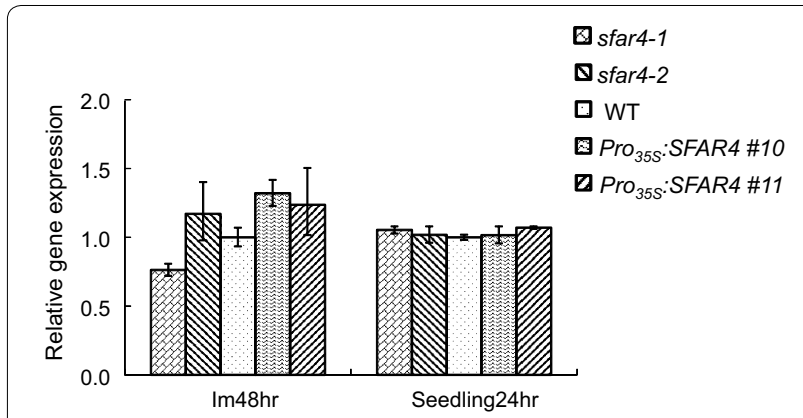

Fig. 4 The relative transcription levels of HXK1 in imbibed seeds (Im48hr) and post-germinated seedlings (24 h). The transcripts were determined by real-time PCR and normalized against actin. The transcription levels are relative to the WT set at level 1. sfar4-1 and sfar4-2 are knockout mutants; WT, wild type; Pro 355 :SFAR4 \#10 and Pro $_{35}$ :SFAR4 \#11 are SFAR4 overexpressors. Each value is the mean of three independent measures, and the error bars correspond to the standard error. The statistical analysis of HXK1 expression in wild type, sfar4 knockout mutants and SFAR4 overexpressors was performed by Student's $t$ test 


\section{SFAR regulates downstream metabolic genes for peroxisomal $\beta$-oxidation}

It has been reported that SFAR4 reduces fatty acid storage and composition in developing seeds (Chen et al. 2012). However, how the gene works remains unclear. To investigate the cause of fatty acid reduction, we detected the expression of the regulatory genes that control fatty acid hydrolysis. The peroxisomal $\beta$-oxidation of fatty acids is a process that plays a crucial role in storage oil mobilization to support seedling establishment in oilseed plants, such as Arabidopsis thaliana. Fatty acids are metabolized through $\beta$-oxidation and converted to sucrose for supplying metabolic energy and carbon skeletons during postgerminative growth. Many mutants with a functional loss of $\beta$-oxidation that thereby diminishes seed germination or seedling growth have been studied. The ped1 (peroxisome defective) mutant lacks thiolase activity required for $\beta$-oxidation. Because of weak fatty acid $\beta$-oxidation, ped1 mutant plants cannot expand their leaves in the absence of sucrose as a result of reduced production of sucrose from storage lipids (Hayashi et al. 1998). CTS transports fatty acids into peroxisomes, and LACS6 and
LACS7 deliver $\beta$-oxidation substrates (Footitt et al. 2002; Fulda et al. 2004). Both are required for storage oil breakdown for post-germinative seedling establishment. CTS regulates acyl CoA transport into the peroxisomes for $\beta$-oxidation and is a major control point for the switch between the opposing developmental programs of dormancy and germination (Footitt et al. 2002). To investigate the relationship between SFAR4 and metabolic genes involved in $\beta$-oxidation, $C T S$ and long-chain acyl-CoA synthetases, LACS6 and LACS7, involved in $\beta$-oxidation were examined. PED1 encoding a 3-ketoacyl-CoA thiolase required for $\beta$-oxidation was also examined. The results showed that SFAR4 raised the expression levels of CTS, LACS6, LACS7, and PED1 in imbibed and germinating seeds (Fig. 5). Suppressing importation of peroxisomal ATP inhibited fatty acid breakdown during early seedling growth. Repressing peroxisomal adenine nucleotide carriers, PNC1 and PNC2, impaired fatty acid breakdown (Linka et al. 2008). We also detected PNC1 and PNC2 expression in wild-type and SFAR4 transgenic plants in this study. The analysis of the transcription levels indicated that SFAR4 upregulated PNC1 but not PNC2

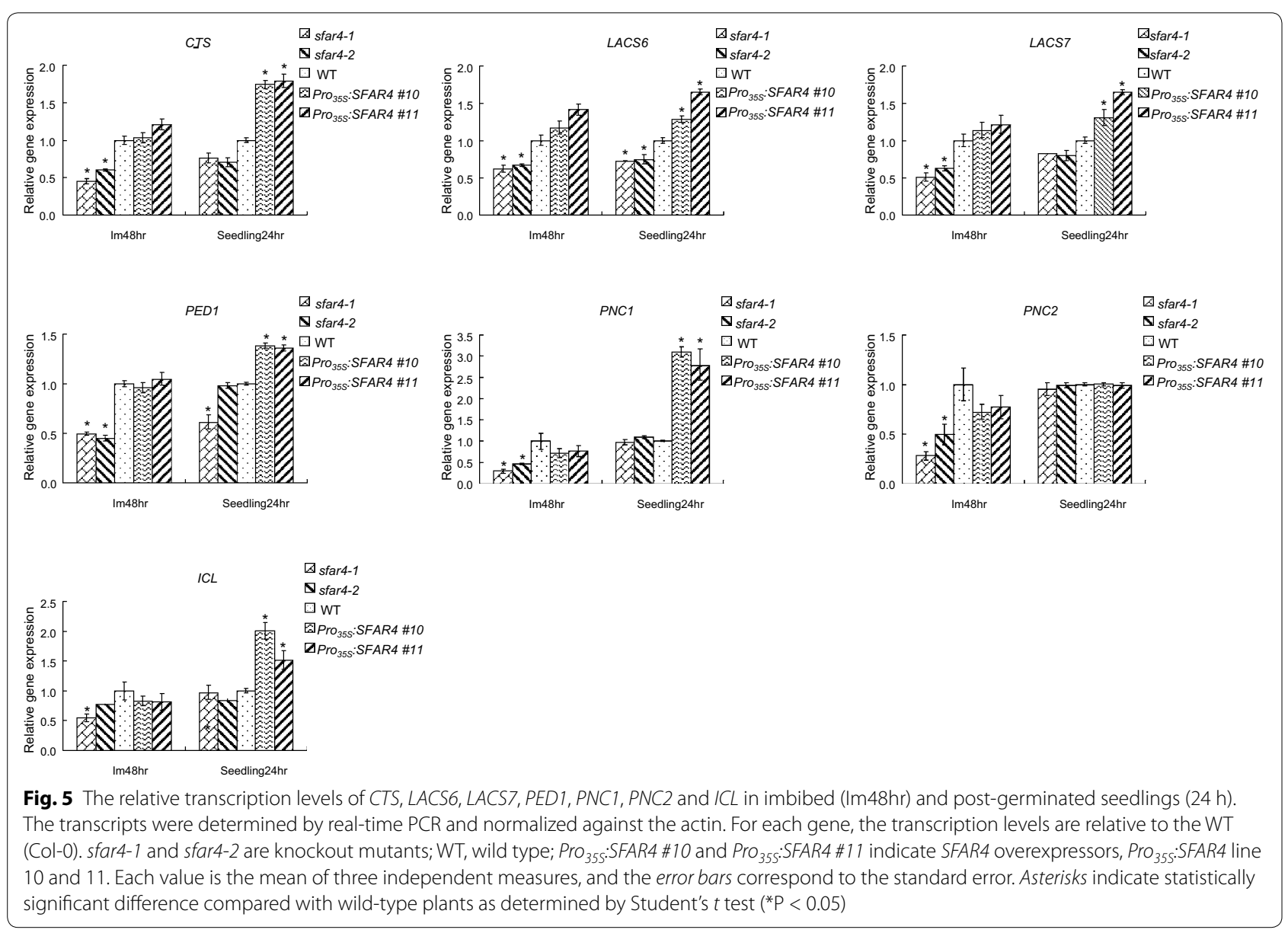


during seedling development (Fig. 5). The $\beta$-oxidation of fatty acids produces acetyl-CoA, which is then converted to sucrose through the glyoxylate cycle and gluconeogenesis. The $\beta$-oxidation substrates were converted into sucrose through the dependent or independent glyoxylate cycle. The glyoxylate cycle is not critical, and an alternative mechanism exists that allows the utilization of fatty acids in plants. Therefore, the glyoxylate cycle is not essential for seed germination (Eastmond et al. 2000). However, it is considered essential for post-germinative growth and seedling establishment in oilseed plants (Eastmond et al. 2000). To elucidate the relationship between SFAR4 and the glyoxylate cycle, ICL, the key factor of the glyoxylate cycle, was evaluated (Fig. 5). A comparison of ICL expression between the Pro $_{35 S}$ SFAR4 transgenic and wild-type plants indicated that SFAR4 elevated ICL during the post-germination stage; however, no significant differences were observed in the imbibed seeds (Fig. 5). This study demonstrated that the gene expression of the glyoxylate cycle key enzyme ICL was regulated by SFAR4 during embryo protrusion rather than in imbibed seeds (Fig. 5). We suggest that SFAR4 reduced fatty acid content by up-regulating $\beta$-oxidation and the glyoxylate cycle during post-germinative growth and seedling establishment.

\section{Conclusions}

In this study, we found that SFAR4 is a GDSL-type esterase that hydrolyzes short-length esters and suggested that SFAR4 requires post-translational modifications for attaining its enzymatic activity. This enzyme played a role in regulating fatty acid degradation through $\beta$-oxidation by up-regulating the expression of CTS, LACS6, LACS7, and PNC1 but not PNC2. SFAR4 overexpressors appear to induce tolerance to glucose during germination and seedling growth, and these studies suggest that SFAR4 may play a crucial role in glucose-regulated pathways or glucose metabolism but not $H X K 1$-dependent pathways.

\section{Additional files}

Additional file 1: Table S1. Primers of PCR and RT-PCR.

Additional file 2: Figure S1. The genomic organization of SFAR4, and primary sequence and structure for SFAR4 protein. (A) Genomic organization of the SFAR4 gene. (B) Protein sequence of SFAR4 deduced from the CDNA sequence. GDSL motif is indicated by the square. Catalytic triad sites Ser45, Asp 187, and His347 are indicated by asterisks.

Additional file 3: Figure S2. Phylogenetic analysis of SFAR4 protein sequence and 24 plant GDSL lipase/esterases elucidated by Mega 4.0 with ClustalW and the NJ method with 1,000 bootstrap replicates. The nodes with less than $50 \%$ bootstrap support are not reported.

Additional file 4: Figure S3. The enzyme activity assay of recombinant SFAR4 proteins. (A) Recombinant SFAR4 proteins were expressed in Pichia pastoris (SMD1168). Secreted proteins were purified and separated by native PAGE and stained with Coomassie Blue. (B) The enzyme activity was determined by a-naphthyl butyrate. Lanes 1, 3, and 5 are the SFAR4 transformants. Lanes 2 and 4 are vector controls. Lanes 1 and 2 are the unconcentrated culture medium ( $20 \mu \mathrm{L} / \mathrm{lane}$ ), while lanes 3 and 4 are concentrated solutions from the culture medium ( $10 \mu \mathrm{g} / \mathrm{lane})$. Lane 5 shows purified recombinant SFAR4 proteins purified with HisTrap Ni Sepharose column from the concentrated solution (1 $\mu \mathrm{g} / \mathrm{lane}$ ).

Additional file 5: Figure S4. Protein motif structure and location of DELLA downregulated GDSL- type enzymes in Arabidopsis. Each colored box represents a particular motif. Their consensus sequence, accession numbers of DELLA regulated GDSL esterase/lipase proteins, and E-value are shown in the left frame. At3G48460 is SFAR4 as presented as a square box.

Additional file 6: Figure S5. Genotype analysis of SFAR4 transgenic plants. (A) Genomic organization of SFAR4 and location of the T-DNA insertion in sfar4-1 and sfar4-2. The arrows indicate the positions of the T-DNA insertions (triangles). Genomic DNA of SFAR4 is represented by 5'UTR, exons (black), introns (white) and 3'UTR. The T-DNA orientation of the left borders (LB) is indicated by the arrow. Chr3 refers to chromosome 3. (B) Southern blot analysis of the T-DNA insertion numbers in Col-0 and sfar4 mutants. The genomic DNA was digested with Hindlll, and the blot was probed with the T-DNA-specific DNA. (C) RNA analysis of SFAR4 gene expression in sfar4-1, sfar4-2, and Col-0. SFAR4 transcript expression was analyzed by RT-PCR. Total RNA $(0.08 \mu \mathrm{g})$ was used to detect SFAR4 and Actinl (loading control) expression. (D) SFAR4 proteins were detected by western blot with specific anti-SFAR4 antibody. RuBisCO was used as a loading control. The quantitative values were tested by Image J free software to analyze and quantify the intensity bands in western blot result images.

Additional file 7: Table S2. Accession numbers of 24 plant GDSL esterases/lipases.

Additional file 8: Figure S6. Phenotype of SFAR4 transgenic plants. (A) Germination rates of SFAR4 transgenic plants in MS medium with or without $10 \mathrm{mM} \mathrm{LiCl}, 150 \mathrm{mM} \mathrm{KCl}$, or $200 \mathrm{mM} \mathrm{NaCl}$. Each value is the mean of three independent measures, and the error bars correspond to the standard error $(n=50)$. (B) Images of Col-0 and SFAR4 transgenic plants in MS medium with or without $10 \mathrm{mM} \mathrm{LiCl}$ for 14 days. sfar4-1 and sfar4-2 are knockout mutants; WT, wild type; Pro ${ }_{35}$ :SFAR4 \#10 and Pro ${ }_{355}:$ SFAR4 \#11 indicate SFAR4 overexpressors, Pro $_{35}$ s:SFAR4 line 10 and 11 . WT is wild type (Col-0).

\section{Abbreviations}

GDSL: GDSL-motif; SFAR: seed fatty acid reducer; CTS: COMATOSE; HXK: hexokinase; GA: gibberellic acid; MS: malate synthase; ICL: isocitrate lyase.

\section{Authors' contributions}

L-MH performed all biochemical and molecular biology experiments, and wrote the paper; J-FS and C-PL supervised the research design and wrote the paper; M-TC and L-FOC performed bioinformatics analysis and plant materials. All authors read and approved the final manuscript.

\section{Author details}

${ }^{1}$ Institute of Biotechnology, National Cheng Kung University, No. 1, University Road, Tainan City 701 , Taiwan. ${ }^{2}$ Department of Food and Beverage Management, Far East University, No. 49, Zhonghua Rd., Xinshi Dist., Tainan City 74448, Taiwan. ${ }^{3}$ Institute of Plant and Microbial Biology, Academia Sinica, No. 128, Sec. 2, Academia Road, Nankang, Taipei 115, Taiwan. ${ }^{4}$ Department of Biological Science and Technology, I-Shou University, No. 1, Sec. 1, Syuecheng Rd., Dashu District, Kaohsiung City 84001, Taiwan. ${ }^{5}$ Agriculture Biotechnology Research Center, Academia Sinica, No. 128, Sec. 2, Academia Road, Nankang, Taipei 115, Taiwan. ${ }^{6}$ Academia Sinica Biotechnology Center in Southern Taiwan, Academia Sinica, No. 59, Siraya Blvd., SinShih Dist., Tainan 74145, Taiwan. ${ }^{7}$ Agricultural Biotechnology Center, National Chung Hsing University, 250 Kuo Kuang Rd., Taichung, Taichung 402, Taiwan.

\section{Acknowledgements}

This research was supported by a grant from the National Science Council of Taiwan number NSC 102-2313-B-269-001 to C. P. Lai. 


\section{Competing interests}

The authors declare that they have no competing interests.

Received: 2 September 2015 Accepted: 16 November 2015 Published online: 01 December 2015

\section{References}

Akoh CC, Lee GC, Liaw YC, Huang TH, Shaw JF (2004) GDSL family of serine esterases/lipases. Prog Lipid Res 43:534-552

Beisson F, Gardies AM, Teissere M, Ferte N, Noat G (1997) An esterase neosynthesized in post-germinated sunflower seeds is related to a new family of lipolytic enzymes. Plant Physiol Biochem 35:761-765

Bewley JD (1997) Seed germination and dormancy. Plant Cell 9:1055-1066

Brick DJ, Brumlik MJ, Buckley JT, Cao JX, Davies PC, Misra S, Tranbarger TJ, Upton C (1995) A new family of lipolytic plant enzymes with members in rice, arabidopsis and maize. FEBS Lett 377:475-480

Cao D, Cheng H, Wu W, Soo HM, Peng J (2006) Gibberellin mobilizes distinct DELLA-dependent transcriptomes to regulate seed germination and floral development in Arabidopsis. Plant Physiol 142:509-525

Cardenas F, Alvarez E, de Castro-Alvarez MS, Sanchez-Montero JM, Valmaseda M, Elson SW, Sinisterra JV (2001) Screening and catalytic activity in organic synthesis of novel fungal and yeast lipases. J Mol Catal B Enzym 14:111-123

Chang SW, Lee GC, Shaw JF (2006) Codon optimization of Candida rugosa lip1 gene for improving expression in Pichia pastoris and biochemical characterization of the purified recombinant LIP1 lipase. J Agric Food Chem 54:815-822

Chen MX, Du X, Zhu Y, Wang Z, Hua SJ, Li ZL, Guo WL, Zhang GP, Peng JR, Jiang LX (2012) Seed fatty acid reducer acts downstream of gibberellin signalling pathway to lower seed fatty acid storage in Arabidopsis. Plant Cell Environ 35:2155-2169

Cho YH, Yoo SD, Sheen J (2006) Regulatory functions of nuclear hexokinase 1 complex in glucose signaling. Cell 127:579-589

Clauss K, Baumert A, Nimtz M, Milkowski C, Strack D (2008) Role of a GDSL lipase-like protein as sinapine esterase in Brassicaceae. Plant J 53:802-813

Clauss K, von Roepenack-Lahaye E, Bottcher C, Roth MR, Welti R, Erban A, Kopka J, Scheel D, Milkowski C, Strack D (2011) Overexpression of sinapine esterase BnSCE3 in oilseed rape seeds triggers global changes in seed metabolism. Plant Physiol 155:1127-1145

Clough SJ, Bent AF (1998) Floral dip: a simplified method for Agrobacteriummediated transformation of Arabidopsis thaliana. Plant J 16:735-743

Cummins I, Edwards R (2004) Purification and cloning of an esterase from the weed black-grass (Alopecurus myosuroides), which bioactivates aryloxyphenoxypropionate herbicides. Plant J 39:894-904

Dalrymple BP, Cybinski DH, Layton I, McSweeney CS, Xue GP, Swadling YJ, Lowry JB (1997) Three Neocallimastix patriciarum esterases associated with the degradation of complex polysaccharides are members of a new family of hydrolases. Microbiology Uk 143:2605-2614

Eastmond PJ, Germain V, Lange PR, Bryce JH, Smith SM, Graham IA (2000) Postgerminative growth and lipid catabolism in oilseeds lacking the glyoxylate cycle. Proc Natl Acad Sci USA 97:5669-5674

Fait A, Angelovici R, Less H, Ohad I, Urbanczyk-Wochniak E, Fernie AR, Galili $G$ (2006) Arabidopsis seed development and germination is associated with temporally distinct metabolic switches. Plant Physiol 142:839-854

Footitt S, Slocombe SP, Larner V, Kurup S, Wu YS, Larson T, Graham I, Baker A, Holdsworth M (2002) Control of germination and lipid mobilization by COMATOSE, the Arabidopsis homologue of human ALDP. EMBO J 21:2912-2922

Footitt S, Marquez J, Schmuths H, Baker A, Theodoulou FL, Holdsworth M (2006) Analysis of the role of COMATOSE and peroxisomal beta-oxidation in the determination of germination potential in Arabidopsis. J Exp Bot 57:2805-2814

Fulda M, Schnurr J, Abbadi A, Heinz E, Browse J (2004) Peroxisomal Acyl-CoA synthetase activity is essential for seedling development in Arabidopsis thaliana. Plant Cell 16:394-405

Graham IA, Eastmond PJ (2002) Pathways of straight and branched chain fatty acid catabolism in higher plants. Prog Lipid Res 41:156-181

Graham IA, Denby KJ, Leaver CJ (1994) Carbon catabolite repression regulates glyoxylate cycle gene expression in cucumber. Plant Cell 6:761-772
Hayashi M, Toriyama K, Kondo M, Nishimura M (1998) 2,4-dichlorophenoxybutyric acid-resistant mutants of Arabidopsis have defects in glyoxysomal fatty acid beta-oxidation. Plant Cell 10:183-195

Holdsworth MJ, Finch-Savage WE, Grappin P, Job D (2008) Post-genomics dissection of seed dormancy and germination. Trends Plant Sci 13:7-13

Hong JK, Choi HW, Hwang IS, Kim DS, Kim NH, du Choi S, Kim YJ, Hwang BK (2008) Function of a novel GDSL-type pepper lipase gene, CaGLIP1, in disease susceptibility and abiotic stress tolerance. Planta 227:539-558

Jang JC, Leon P, Zhou L, Sheen J (1997) Hexokinase as a sugar sensor in higher plants. Plant Cell 9:5-19

Karve A, Xia X, Moore B (2012) Arabidopsis hexokinase-like1 and hexokinase1 form a critical node in mediating plant glucose and ethylene responses. Plant Physiol 158:1965-1975

Kelly AA, Quettier AL, Shaw E, Eastmond PJ (2011) Seed storage oil mobilization is important but not essential for germination or seedling establishment in Arabidopsis. Plant Physiol 157:866-875

Koch K (2004) Sucrose metabolism: regulatory mechanisms and pivotal roles in sugar sensing and plant development. Curr Opin Plant Biol 7:235-246

Li J, Derewenda U, Dauter Z, Smith S, Derewenda ZS (2000) Crystal structure of the Escherichia coli thioesterase II, a homolog of the human Nef binding enzyme. Nat Struct Biol 7:555-559

Ling H, Zhao J, Zuo K, Qiu C, Yao H, Qin J, Sun X, Tang K (2006) Isolation and expression analysis of a GDSL-like lipase gene from Brassica napus L. J Biochem Mol Biol 39:297-303

Linka N, Theodoulou FL, Haslam RP, Linka M, Napier JA, Neuhaus HE, Weber APM (2008) Peroxisomal ATP import is essential for seedling development in Arabidopsis thaliana. Plant Cell 20:3241-3257

Mikleusevic G, Salopek-Sondi B, Luic M (2009) Arab-1, a GDSL lipase from the model plant, Arabidopsis thaliana (L.) Heynh. Croat Chem Acta 82:439-447

Molgaard A, Kauppinen S, Larsen S (2000) Rhamnogalacturonan acetylesterase elucidates the structure and function of a new family of hydrolases. Structure 8:373-383

Moore B, Zhou L, Rolland F, Hall Q, Cheng WH, Liu YX, Hwang I, Jones T, Sheen $J$ (2003) Role of the Arabidopsis glucose sensor HXK1 in nutrient, light, and hormonal signaling. Science 300:332-336

Pego JV, Weisbeek PJ, Smeekens SC (1999) Mannose inhibits Arabidopsis germination via a hexokinase-mediated step. Plant Physiol 119:1017-1023

Penfield S, Rylott EL, Gilday AD, Graham S, Larson TR, Graham IA (2004) Reserve mobilization in the Arabidopsis endosperm fuels hypocotyl elongation in the dark, is independent of abscisic acid, and requires PHOSPHOENOLPYRUVATE CARBOXYKINASE1. Plant Cell 16:2705-2718

Poirier Y, Ventre G, Caldelari D (1999) Increased flow of fatty acids toward betaoxidation in developing seeds of Arabidopsis deficient in diacylglycerol acyltransferase activity or synthesizing medium-chain-length fatty acids. Plant Physiol 121:1359-1366

Richards DE, King KE, Ait-ali T, Harberd NP (2001) How gibberellin regulates plant growth and development: a molecular genetic analysis of gibberellin signaling. Ann Rev Plant Physiol Plant Mol Biol 52:67-88

Rolland F, Baena-Gonzalez E, Sheen J (2006) Sugar sensing and signaling in plants: conserved and novel mechanisms. Ann Rev Plant Biol 57:675-709

Ruppert M, Woll J, Giritch A, Genady E, Ma X, Stockigt J (2005) Functional expression of an ajmaline pathway-specific esterase from Rauvolfia in a novel plant-virus expression system. Planta 222:888-898

Schoof H, Lenhard M, Haecker A, Mayer KFX, Jurgens G, Laux T (2000) The stem cell population of Arabidopsis shoot meristems is maintained by a regulatory loop between the CLAVATA and WUSCHEL genes. Cell 100:635-644

Smeekens S (2000) Sugar-induced signal transduction in plants. Ann Rev Plant Physiol Plant Mol Biol 51:49-81

Solanas M, Escrich E (1997) An improved protocol to increase sensitivity of Southern blot using dig-labelled DNA probes. J Biochem Biophys Methods 35:153-159

Sun TP, Gubler F (2004) Molecular mechanism of gibberellin signaling in plants. Ann Rev Plant Biol 55:197-223

To JP, Reiter WD, Gibson SI (2002) Mobilization of seed storage lipid by Arabidopsis seedlings is retarded in the presence of exogenous sugars. BMC Plant Biol 2:4

Upton C, Buckley JT (1995) A new family of lipolytic enzymes? Trends Biochem Sci 20:178-179

Winnepenninckx B, Backeljau T, De Wachter R (1995) Phylogeny of protostome worms derived from 18S rRNA sequences. Mol Biol Evol 12:641-649 